\title{
Diphlorethohydroxycarmalol of Ishige okamurae and Caffeine Modified the Expression of Extracellular Fibrillars during Adipogenesis of Mouse Subcutaneous Adipose Derived Stem Cell
}

\author{
Younmi Jeon ${ }^{1}$, Siyoung Song ${ }^{2}$, Hagju $\mathrm{Kim}^{3}$ and ${ }^{\dagger}{ }^{\dagger}$ ong-Pil Cheon ${ }^{1}$ \\ ${ }^{1}$ Division of Developmental Biology and Physiology, School of Biosicences and Chemistry, \\ Sungshin Women's University, Seoul 142-742, Republic of Korea \\ ${ }^{2}$ NSTECH Co. Ltd., Incheon 405-848, Republic of Korea \\ ${ }^{3}$ Seojin BioTech Co. Ltd., Suwon 443-373, Republic of Korea
}

\begin{abstract}
Although, one of the etiologies of localized lipodystrophy of the subcutaneous connective tissue (cellulite) is the histological alternation of adipose tissue, the characteristics of expression of the components of extracellular matrix (ECM) components during adipogenesis are not uncovered. In this study, the effects of caffeine and Ishige okamurae originated diphlorethohydroxycarmalol (DPHC) on the expression of extracellualr fibers was analyzed with quantitative RT-PCR during differentiation induction of mouse subcutaneous adipose derived stem cells (msADSC) into adipocyte. The expression levels of Col1a, Col3a1, and Col61a were decreased by the adipogenci induction in a time-dependent manners. However, Col2a mRNA and Col4a1 mRNA expressions were oposit to them. Caffeine and DPHC stimulated the changes of the expression of these collagens. Eln mRNA expression was increased by induction. DPHC stimulated the expression of it. Mfap5 mRNA expression was deceased in both adipogenic cell and matured adipocytes. Caffeine suppressed the expression of Mfap5 but the effect of DPHC was different by the concentration. The expression of bioglycan, decorin, and lumican were also modified by caffeine and DPHC in a concentration-dependent manner. Based on this study, we revealed firstly the effects of caffeine and DPHC on the expression of collagens, elastin, and glycoproteins during adipogenesis of msADSCs. Those results suggest that DPHC may have antiadipogenic effect and has more positive effets on normal adipose tissue generation and work as suppressor the abnormality of ECM structure. Such results indicate that DPHC can be applied in keeping the stability of the ECM of adipogenic tissues.
\end{abstract}

Key words : Mouse subcutaneous adipose derived stem cell, Diphlorethohydroxycarmalol, Extracellular matrix, Extracellular fibril, Cellulite, Caffeine, Adipogenesis

\section{INTRODUCTION}

Mesenchymal cells form adipose tissues in various histological areas during embryo development with various purposes. The physiological roles of these adipose tissues are different by their anatomical sites (Cinti, 2001) and the adipose tissues are under the pattern specification (Gesta et al., 2006; Perrini et al., 2008). Histological changes in dermo-hypodermic tissue are the cause of deformation of the integument. Cellulite is a typical example of deformation of the integument. Such structural changes affect $80-90 \%$ of adult female (Pavicic et al., 2006) and has various

\footnotetext{
Manuscript received 2 September 2013, Received in revised form 6 September 2013, Accepted 13 September 2013

† Corresponding Author : Yong-Pil Cheon, Division of Developmental Biolgoy and Physiology, School of Biosicences and Chemistry, Sungshin Women’s University, Mia-dong, Kangbuk-gu, Seoul, 142-742, Korea. Tel. : +82-2-920-7639, Fax : +82-2-920-2093, E-mail : ypcheon@sungshin.ac.kr

This is an Open Access article distributed under the terms of the Creative Commons Attribution Non-Commercial License(http://creativecommons.org/ licenses/by-nc/3.0) which permits unrestricted non-commercial use, distribution, and reproduction in any medium, provided the original work is properly cited.
} 
names such as adiposis edematosa, lipodystrophy, edematofibrosclerotic panniculitis, liposclerosis, dermopanniculosis deformans, status protrusus cutis, gynoid lipodystrophy, nodular liposclerosis, and orange peel syndrome (de la Casa Almeida et al., 2012; Milani et al., 2008). Cellulite is the herniation of subcutaneous fat within fibrous connective tissue that manifests topographically as skin dimpling and nodularity, often on the pelvic region, lower limbs, and abdomen (de la Casa Almeida et al., 2012; Rossi \& Vergnanini, 2000).

It is suggested that cellulite is different from obesity because adipocyte hypertrophy and hyperplasia is appeard both of them in physiological status wherease there is structural alterations of the dermis only in cellulite which is accompanied with additional morphological, histochemical, biochemical and ultrastructural modification in cellulite (Bray, 1989; Curri, 1983; de la Casa Almeida et al., 2012). The increase the number of adipocytes and the growth of the adipocyte is primary condition for cellulite. During adipose growth, the extracellular matrix requires remodeling (Khan et al., 2009). It has been detected that women with high BMI and cellulite have weaker and less dense connective tissue in integument (Mirrashed et al., 2004; Ortonne et al., 2008). Therefore, the alternation of the connective tissue and the increase of the number of adipocytes are suspected as a major physiological factor for cellulite.

Increase the size of fat tissue is depending on the growth of adipocyte and hyperplasia of adipose stem cells. Accumulation of the excess energy into fat cell is charicterized by an increase in white fat. By the accumulation or dissociation of white mass, the adipose tissue has to remodel for reconstruct the characteristic histology. It is associated with remodeling of the extracellular matrix (ECM) and angiogenesis (Hausman \& Richardson, 2004). Primarily the ECM in adipose tissue supports the structure of adipocytes and other cells. It also participates in a various events in cells (Babelova et al., 2009). Production of ECM proteins and remodeling proteases such as matrix metalloprotease and their inhibitors (TIMPs), and the angiogenic factors is the mediator for the remodeling (Cao et al., 2001; Chavey et al., 2003; Chiellini et al., 2003; Chun et al., 2006).

The health of cutaneous is important to keep the structure. There are various treatment models for the disformation of cutaneous. One of the approaches to treatment is the regulation of expression of specific genes. It is currently fully uncovered which extracellular fibril genes are expressed during such a dermal disformation. It has been reported that acid proteoglycans and 2-macroglobulin are abundant in the dimpling skin area (Agache et al., 1976). During adipogenesis a few of extracellular fibris are expressed. Their expressions are effected by the varoius stimulation such as metabolic stress (Khan et al., 2009). Numerical pharmacologic agents such as aminophylline and herbal products have been used to prevent or cure the cellulite. The expected main results of these are decrease the size of adipose tissue (Khan et al., 2010). It has been suggested that the control of expression or modulation of extracellular fibrils is a new approach to suppress the structural changes in cutaneous.

Diphlorethohydroxycarmalol (DPHC) of Ishige okamurae is suggested as an antioxidant (Heo et al., 2009) and inducing substance of apoptosis in 3-T3-L1 preadipocyte (Park et al., 2013). DPHC also stimulates the expression of cyclooxygenase (COX)- 1 and COX-2 in both levles of transcription and translation in HaCaT human cell (Kang et al., 2012). To understanding the adipogenesis and cellulite, it is important to understanding the expression of ECM fibrils. Put together with the prementioned physiological role of extracellular fibrils and the increase of adipose tissue in the changes of cutaneous, we examined in this study, the effects of DPHC in the expression of extracellular fibrils during adipogenesis of subcutaneous adipose derived stem cells.

\section{MATERIALS AND METHODS}

\section{Isolation of diphlorethohydroxycarmalol}

DPHC was isolated at Seojin Coorporation according to the established method (Heo et al., 2009). Briefly, the dried I. okamurae was extracted three times with $80 \%$ methanol and filtered. Then the filtrate was under evaporation 
DPHC and Caffeine on ECM Fibrillar Expression in msADSC Adipogenesis

at $40^{\circ} \mathrm{C}$. The I. okamurae methanol extract was suspended on distilled water and the partitioned with ethyl acetate. The ethyl acetate fraction was subjected to silica gel and Sephadex-LH 20 column chromatography. The DPHC was purified by high performance liquid chromatography (HPLC) using a Waters HPLC system equipped with a Waters 996 photodiode array detector and C18 column (J'sphere ODS-H80, 150×20 mm, 4 ; YMC Co.) by stepwise elution with acetonitrile-water gradient (UV range: $230 \mathrm{~nm}$, flow rate: 0.8 /min). Finally, the structure of the DPHC was identified by comparing the NMR spectral data with those in existing literature.

\section{Mouse subcutaneous adipose-derived stem cells} (msADSC) isolation and culture

All experimental animal studies followed to the Guide for the Care and Use of Laboratory. Animals were maintained under standard conditions at Sungshin Women's University with 14 hour light - 10 hour dark light cycle. Animals were fed a standard rodent diet and water ad libitum from weaning at 21 days of age. Mouse subcutaneous adipose tissue was obtained from the CD-1 female mice (10 - 12 weeks old). In briefly, approximately $2 \mathrm{~g}$ of mouse subcutaneous adipose tissue was washed several times in Hank's buffered salt solution (HBSS), containing 1\% BSA, $200 \mathrm{nM}$ adenosine, and $50 \mathrm{mg} /$ glucose. The adipose tissue was minced finely using scissors and incubated in digestion buffer at $37^{\circ} \mathrm{C}$ with constant agitation for 1 hour. The digestive buffer contained 0.1\% type I collagenase (Gibco, Cat\# 17100-017) and 1\% bovine serum albumin. After digestion the mononuclear cells were washed and seeded. These mouse subcutaneous adipose derived stem ells (msADSCs) were cultured in Dulbecco's modified Eagle's medium-low glucose (DMEMLG) (Gibco, Cat\# 31600-026) containing 10\% fetal bovine serum (FBS) (Welgene, Cat\# S001-07), $100 \mathrm{U} /$ penicillin, $0.1 \mathrm{mg} / \quad$ streptomycin, and $3.7 \mathrm{mg} / \quad$ sodium bicarbonate. All of the nucleated cells were plated at 25,000 cells/ density in 10 of medium in a culture dish and incubated at $37^{\circ} \mathrm{C}$ with $5 \% \mathrm{CO}_{2}$.. After $24 \mathrm{hr}$, nonadherent cells were discarded, and adherent cells were thoroughly washed twice with PBS. Medium was changed every other day. To prevent spontaneous differentiation, cells were maintained at subconfluent levels. To evaluate the effects of caffeine and DPHC on the expression of ECM fibrils, sADSCs were cultured in the adipogenic induction media containing caffeine $(0.05 \mathrm{mM}$ and $0.1 \mathrm{mM})$ or DPHC $(0.4 /, 2 /, 10 /$, and $50 /$ ).

1) Induction of adipogenesis of mouse subcutaneous derived adipose stem cells

msADSCs were cultivated in monolayer until 95\% confluence in DMEM supplemented with 10\% fetal bovine serum (FBS) (Welgene, Cat \#. S001-07), 100 U/ penicillin, $0.1 \mathrm{mg} /$ streptomycin, $3.7 \mathrm{mg} /$ sodium bicarbonate at $5 \% \mathrm{CO}_{2}, 37^{\circ} \mathrm{C}$. And then, medium was changed with adipocyte induction medium and cultured for 14 days. The induction media containing $10 \% \mathrm{FBS}, 10 \mu \mathrm{M}$ insulin, $0.5 \mathrm{mM}$ isobutilmethilxantine, $1 \mu \mathrm{M}$ dexamethasone, and $200 \mu \mathrm{M}$ indomethacin. The acquisition of the adipogenic phenotype was determined by staining the monolayers with $2 \%$ Oil Red-O solution.

\section{2) Gene expression analysis}

msADSCs were maintained in noninductive control medium until 90-95\% confluent the culture plate. After adipogenic induction the cells were collected at 8 day and 14 day after induction, respectively to analyze the expression of extracellular fibrils. The expression profiles of the genes for extracellular fibrils genes were analyzed. Total RNAs from cells were isolated using TRIzol Reagent according to the manufacturer's instructions. The purity of RNA was assessed by determining the ratio of absorbance at $260 \mathrm{~nm}$ to that at $280 \mathrm{~nm}$. First strand cDNA was synthesized using First-Strand synthesis system (Stratagene, Cat\# 200420) according to the manufacturer's instructions. Briefly, the mixtures were incubated at $65^{\circ} \mathrm{C}$ for minutes and place tube at room temperature for 10 minutes for the primers to anneal to the RNA. And incubated at $42^{\circ} \mathrm{C}$ for $60^{\circ} \mathrm{C}$ minutes and incubated at $70^{\circ} \mathrm{C}$ 
Table 1. Sequences of sense (S) and antisense (AS) primers (5'-3') for Real-time RT-PCR

\begin{tabular}{|c|c|c|}
\hline Genes & Gene Bank Access No. & Sequences \\
\hline \multirow{2}{*}{ Col1a1 } & \multirow{2}{*}{ NM_007742 } & S: AAACCCTGGTGCTGATGGACAA \\
\hline & & AS: ACCAGTGTCTCCTTTGTTGCCA \\
\hline \multirow{2}{*}{ Col2a1 } & \multirow{2}{*}{ NM_031163 } & S: TGGTGACAAGGGAGAAAAGGGT \\
\hline & & AS: ACCACCAGCCTTCTCGTCATA \\
\hline \multirow{2}{*}{ Col3a1 } & \multirow{2}{*}{ NM_009930 } & S: CTCAСССТТСТТСАТСССАСТСТT \\
\hline & & AS: AGGTGTAGAAGGCTGTGGGCATA \\
\hline \multirow{2}{*}{ Col4a1 } & \multirow{2}{*}{ NM_009931 } & S: CAAAAGGTGACAAGGGAGAGCAAG \\
\hline & & AS: GCTCССССТTТСТССТTTTTСА \\
\hline \multirow{2}{*}{ Col6a1 } & \multirow{2}{*}{ NM_009933 } & S: AGCCAGACCATTGACACCATTG \\
\hline & & AS: TCСССТТTТСТСССТТСАТАСС \\
\hline \multirow{2}{*}{ Bgn } & \multirow{2}{*}{ NM_007542 } & S: TGACAACCGTATCCGCAAAGTG \\
\hline & & AS: AAGTTCGTTCAGGGTCTCAGGGA \\
\hline \multirow{2}{*}{ Eln } & \multirow{2}{*}{ NM_007925 } & S: TCCTCTTGCTCAACСТCСТCСАT \\
\hline & & AS: TGCTCCAAACGTTCCCAGA \\
\hline \multirow{2}{*}{ Dcn } & \multirow{2}{*}{ NM_001190451 } & S: CTCATCTTCTTCСTTCTGGCACA \\
\hline & & AS: ATCCCAGGGCACTTTGTCCA \\
\hline \multirow{2}{*}{ Lum } & \multirow{2}{*}{ NM_008524 } & S: ATGTGCGTTCTCTCTTGCCTT \\
\hline & & AS: ACTTGATGCCAGGAGGAACCA \\
\hline \multirow{2}{*}{ Mfap5 } & \multirow{2}{*}{ NM_015776 } & S: GCTGGTGACAAAAATGCTACTGC \\
\hline & & AS: ACAGGGAGGAAGTCGGAAGTAGTT \\
\hline \multirow{2}{*}{$T g f b 1$} & \multirow{2}{*}{ NM_011577 } & S: ACCGCAACAACGCCATCTAT \\
\hline & & AS: GGTAACGCCAGGAATTGTTGCT \\
\hline
\end{tabular}

for 15 minutes to terminate cDNA synthesis. Quantitative real-time PCR was performed for extracellular fibrils using their specific primers (Table 1).

\section{3) Statistical analysis}

All quantitative real-time PCR data were subjected to two way ANOVA and Student $t$-test. Real-time PCR data were corrected for differences in sample loading using the Arbp data as a covariate. A P value of less than 0.05 was considered significant vs. control and caffeine or DPHC treatment groups.

\section{RESULTS}

1. Characteristics of the expression of collagens during adipogenesis of subcutaneous derived adipose stem cell

Mesenchymal cells express collagens at the time of adi- pogenesis of themselves. In here we analyze the expression of collagens during adipogenesis of msADSCs. During adipogenesis of msADSCs, Col1a mRNA expression was decreased by the induction in the time dependent manner (Fig. 1A). After 14 days of adipogenic induction, the expression levels were significantly lower than that of noninduced msASCs. The mRNA expression patterns of Col3a1 and Col61a were similar with that of Col1a1 mRNA expression (Fig. 3A, Fig. 5A). In the case of Col2a, its mRNA expression is increased by the induction in the time-dependent manner. Its expression levels were significantly high at day 8 and 14 of adipogenic induction (Fig. 2A). Col41a mRNA expression pattern was similar with that of Col2a mRNA expression (Fig. 4A).

\section{Caffeine changes the expression patterns of} collagens during adipogenesis of subcutaneous derived adipose stem cell 

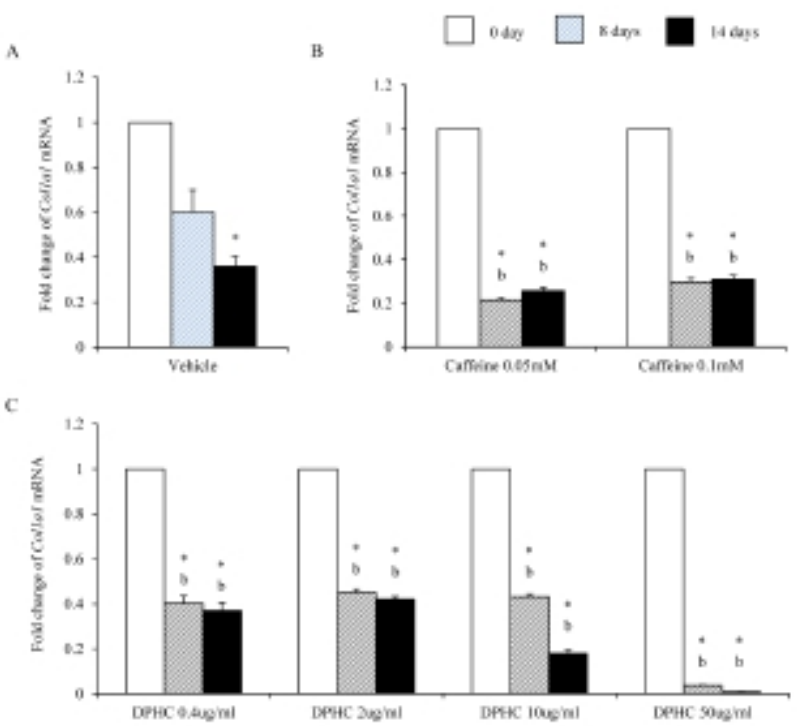

Fig. 1. Effects of caffeine and diphlorethohydroxycarmalol (DPHC) of collagen type 1 gene (Col1a1). Mouse subcutaneous adipose derived stem cells (msADSCs) were incubated until 90-95\% confluency and then induced to adipocyte. Total RNAs were isolated at 0 day, 8 day, and 14 day of induction. To evaluate the change of the expression levels of Col1a1 mRNA, real-time PCR method was employed. *: $P<0.05$ control vs. caffeine or DPHC.
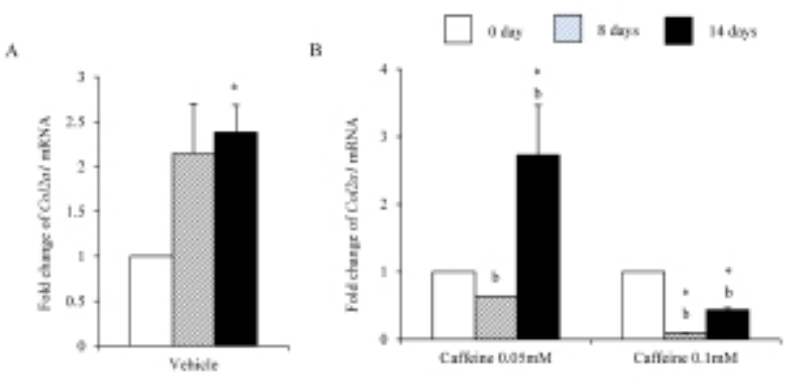

c

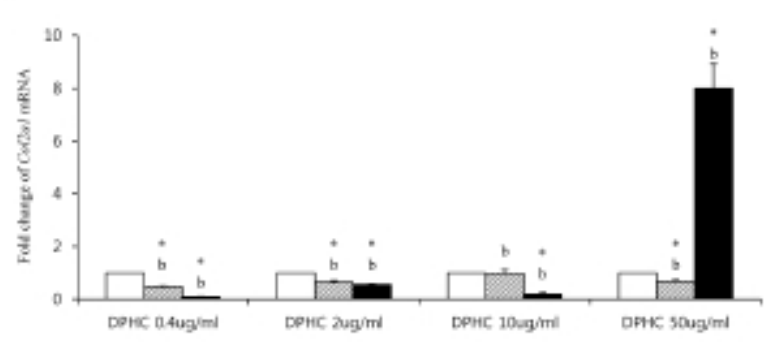

Fig. 2. Effects of caffeine and diphlorethohydroxycarmalol (DPHC) of collagen type 2 gene (Col2a1). Mouse subcutaneous adipose derived stem cells (msADSCs) were incubated until 90-95\% confluency and then induced to adipocyte. Total RNAs were isolated at 0 day, 8 day, and 14 day of induction. To evaluate the change of the expression levels of Col2a1 mRNA, real-time PCR method was employed. *: $P<0.05$ control vs. caffeine or DPHC.

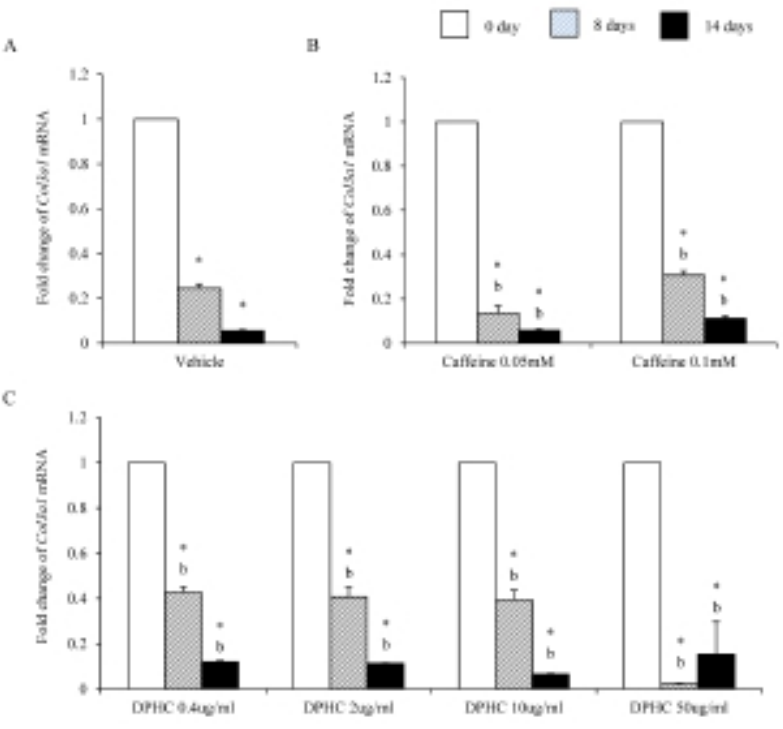

Fig. 3. Effects of caffeine and diphlorethohydroxycarmalol (DPHC) of collagen type 3 gene (Col3a1). Mouse subcutaneous adipose derived stem cells (msADSCs) were incubated until 90-95\% confluency and then induced to adipocyte. Total RNAs were isolated at 0 day, 8 day, and 14 day of induction. To evaluate the change of the expression levels of Col3a1 mRNA, real-time PCR method was employed. *: $P<0.05$ control vs. caffeine or DPHC.
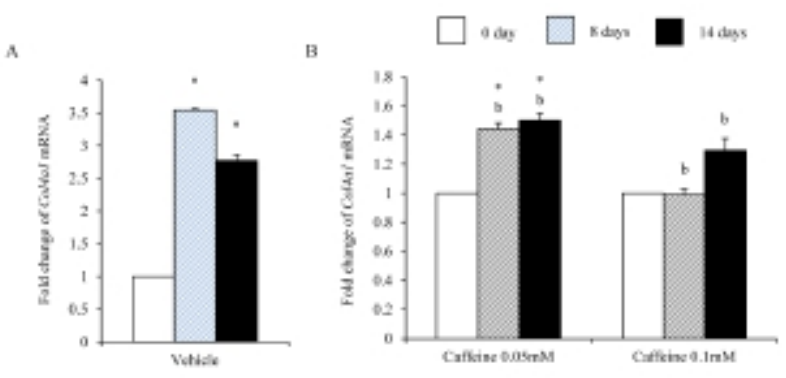

c

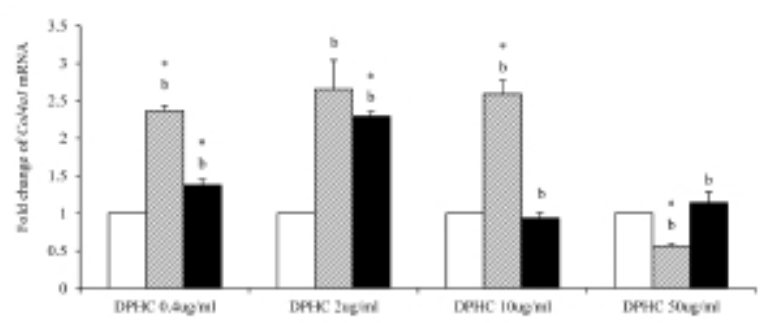

Fig. 4. Effects of caffeine and diphlorethohydroxycarmalol (DPHC) of collagen type 4 gene (Col4a1). Mouse subcutaneous adipose derived stem cells (msADSCs) were incubated until 90-95\% confluency and then induced to adipocyte. Total RNAs were isolated at 0 day, 8 day, and 14 day of induction. To evaluate the change of the expression levels of Col4a1 mRNA, real-time PCR method was employed. *: $P<0.05$ control vs. caffeine or DPHC. 
Caffeine, a methylxanthines, is widely used in cellulite therapy (Khan et al., 2010). Therefoere in this study, it is used as control for DPHC. Col1a1 mRNA expression was dramatically and significantly decreased after adipogenic induction in the all concentration groups as seen in Fig. 1B. The mRNA expression patterns of Col3a1 and Col6a1 were same with that of Col1a1 (Fig. 3B, Fig. 5B). The patterns of mRNA expression of Col2a1 were different from that of Col1a1. Col12a1 mRNA expression was significantly increased by adipogenic induction in 0.05 $\mathrm{mM}$ but its levels were significantly decreased in 0.1 $\mathrm{mM}$ caffeine (Fig. 2B). Col4a1 mRNA expression patterns were similar with that of Col2a1 (Fig. 4B).

Col2a1 mRNA and Col4a1 mRNA expression was suppressed by caffeine in a dose depenent manners compared with the nontreated experiments (Fig. 2, Fig. 3).

\section{DPHC changes the expression patterns of collagens} during adipogenesis of subcutaneous derived adipose stem cell

To know the effects of DPHC on the adipogenesis, the mRNA expressions of collagens were analyzed as mentioned in Materials and Methods. The expression patterns of collagen genes in the DPHC treated groups were various between them. Col1a1 mRNA and Col3a1 mRNA expression was significantly suppressed by DPHC in a concentratio- and induction time-dependent manners (Fig. 1C, Fig. 3C). The expression levels of Col1a1 mRNA were significantly low in 50 / DPHC group on day 15 of induction. The expression patterns during adipogenesis were more similar to vehicle control than caffeine groups (Fig. 1). In Col3a1 mRNA expression, there was no significant difference between caffeine and DPHC in differentiated adipocytes on day 14 of induction (Fig. 3).

Col2a1 mRNA expressions were significantly suppressed by $0.4 /, 2 /$, and $10 /$ DPHC and the adipogenic induction, but its expression was significantly increased in 50 / DPHC after 14 days of adipogenic induction (Fig. 2C). Col4a1 mRNA expression was significantly increased in $0.4 /$ and $2 /$ in induction time dependent manner. In 10 / DPHC, its expression was significantly high on day 8 of induction but its expression was dramatically decreased after then. In 50 / DPHC, its expression was similar with that of vehicle control with except on day 8 of induction. Its expression level was significantly low and recovered to the level of vehicle control on day 14 (Fig. 4C). The tendency of Col6a1 mRNA expression is decrease in the DPHC treatment groups (Fig. 5C). The expression levels of Col2a1 mRNA were significantly high in 0.4-50 / DPHC groups (Fig. 2).

\section{The patterns of elastin mRNA and microfibrilla} associated protein 5 mRNA expressions during adipogenesis and the effects of caffeine and DPHC

Eln mRNA expression was significantly increased until day 8 of induction but after then expression levels decreased in in vitro (Fig. 6A). The expression levels of Eln mRNA were modified by caffeine during adipogenesis. Its expression was not change in $0.05 \mathrm{mM}$ until 14 days of induction. However, its expression was significantly increased in $0.1 \mathrm{mM}$ caffeine group on 8 days of induction. But its expression level was significantly decreased on 14 days of induction. It showed that the level of mRNA expression of Enl is depending on the stage of adipogenesis (Fig. $6 \mathrm{~B})$. In the case of DPHC, it stimulated the mRNA expression of Eln in all concentration groups (Fig. 6C). The expression levels of Eln mRNA were higher than caffeine groups on day 14 of induction (Fig. 6).

It is known that MFAP5 is associated with elastin networks in adipose tissue. Interestingly the expression levels of Mfap5 mRNA were significantly low in both adipogenic cell and matured adipocytes which were differentiated from sADSC (Fig. 7A). Caffeine strongly suppressed the expression of Mfap5 mRNA during induction and after differentiation in all concentration (Fig. 7B). In the case of DPHC, the expression of Mfap5 mRNA was suppressed in all concentration except $0.4 / .0 .4 /$ DPHC suppressed the expression of Mfap5 mRNA during induction but it was not suppressed the expression of Mfap5 mRNA in differentiated adipocytes. There was significantly difference between caffeine 

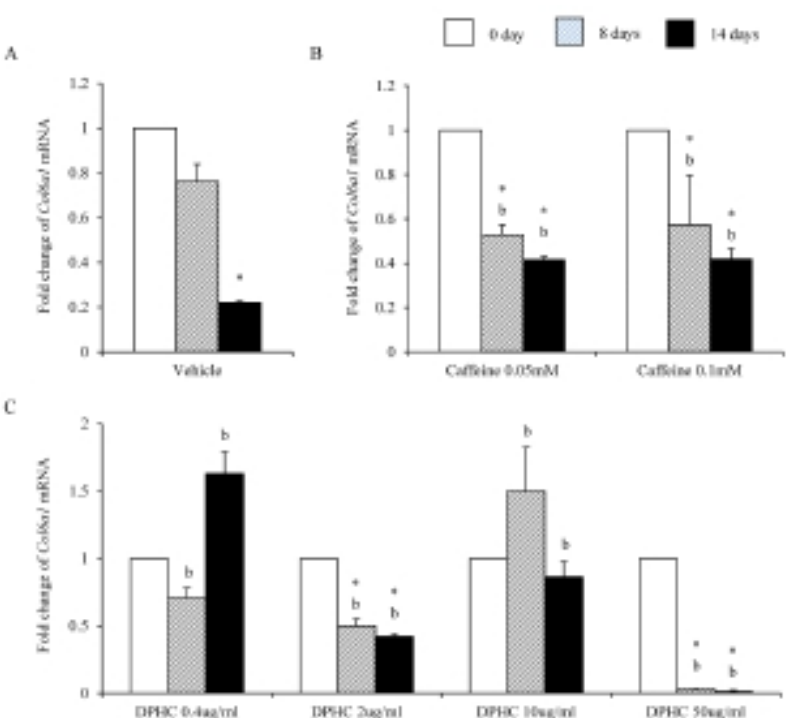

Fig. 5. Effects of caffeine and diphlorethohydroxycarmalol (DPHC) of collagen type 6 gene (Col6a1). Mouse subcutaneous adipose derived stem cells (msADSCs) were incubated until 90-95\% confluency and then induced to adipocyte. Total RNAs were isolated at 0 day, 8 day, and 14 day of induction. To evaluate the change of the expression levels of Col6a1 mRNA, real-time PCR method was employed. *: $P<0.05$ control vs. caffeine or DPHC.
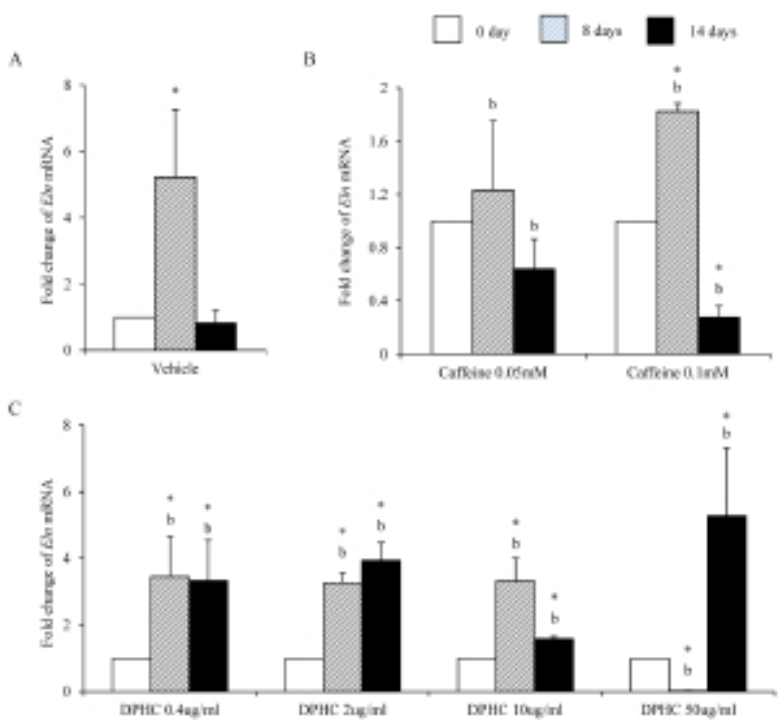

Fig. 6. Effects of caffeine and diphlorethohydroxycarmalol (DPHC) of elastin genes (Ela). Mouse subcutaneous adipose derived stem cells (msADSCs) were incubated until 9095\% confluency and then induced to adipocyte. Total RNAs were isolated at 0 day, 8 day, and 14 day of induction. To evaluate the change of the expression levels of Ela mRNA, real-time PCR method was employed. *: $P<0.05$ control vs. caffeine or DPHC.

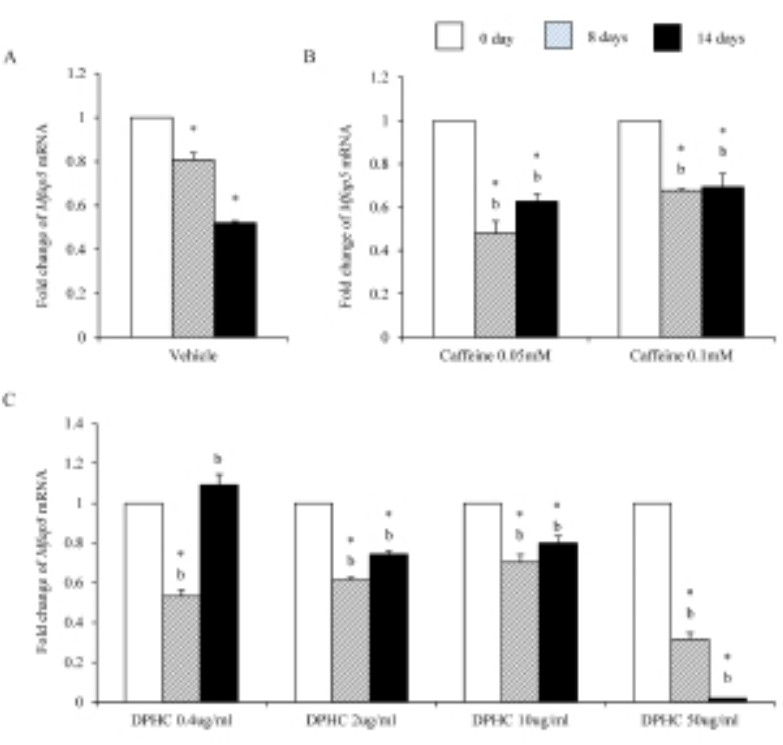

Fig. 7. Effects of caffeine and diphlorethohydroxycarmalol (DPHC) of microfibrilla associated protein 5 gene (Mfap5). Mouse subcutaneous adipose derived stem cells (msADSCs) were incubated until 90-95\% confluency and then induced to adipocyte. Total RNAs were isolated at 0 day, 8 day, and 14 day of induction. To evaluate the change of the expression levels of Mfap5 mRNA, real-time PCR method was employed. *: $P<0.05$ control vs. caffeine or DPHC.
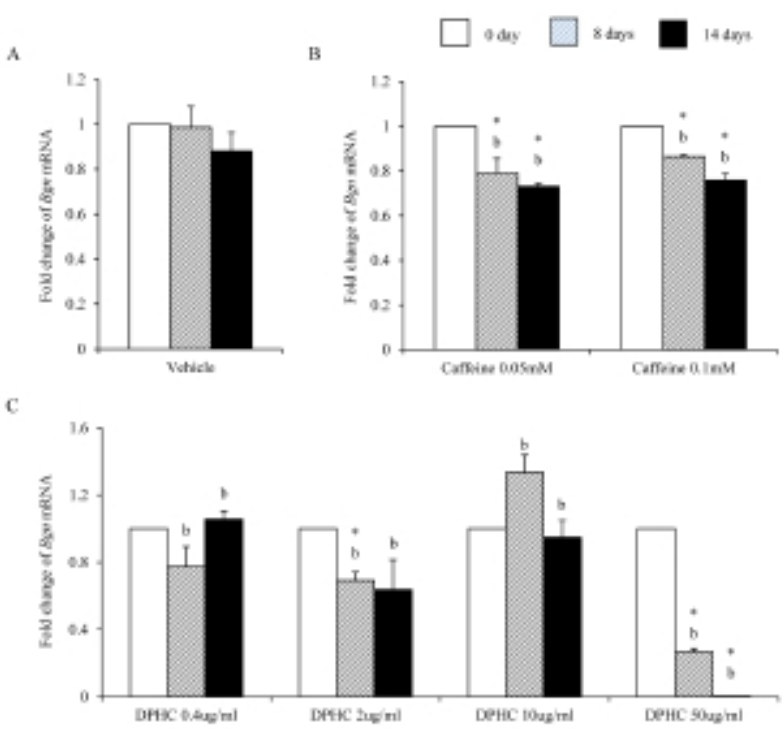

Fig. 8. Effects of caffeine and diphlorethohydroxycarmalol (DPHC) of biglycan gene (Bgn). Mouse subcutaneous adipose derived stem cells (msADSCs) were incubated until 9095\% confluency and then induced to adipocyte. Total RNAs were isolated at 0 day, 8 day, and 14 day of induction. To evaluate the change of the expression levels of Bgn mRNA, real-time PCR method was employed. *: $P<0.05$ control vs. caffeine or DPHC. 
and 50 / DPHC on day 14 of induction (Fig. 7).

\section{The effects of caffeine and DPHC on the expression}

\section{of glycoproteins}

Bioglycan, decorin, and lumican are member of leucinerich proteoglycan. During adipogenesis of SADSC, the expression levels of Bgn mRNA kept same levels with noninduced sADSC (Fig. 8A). However, caffeine suppressed the expression of Bgn mRNA in all concentration (Fig. 8B). The expression of Bgn mRNA was suppressed on 50 / DPHC during adipogenesis. However, in the lower concentration than $50 /$, its expressions were not changed (Fig. 8C). Comparing with caffeine the expression levels were significantly lower in 50 / DPHC on day 14 of induction (Fig. 8).

During adipogenesis of sADSCs, Dcn mRNA expression was significantly decreased by adipogenesis in vehicle control (Fig. 9A). Dcn mRNA expression levels were not changed until day 8 of induction but its expression was significantly decreased on day 14 of adipogenesis in all concentrations (Fig. 9B). In the case of DPHC, the expression levels of Dcn mRNA were significantly increased in 10 / by induction. However, its expressions were suppressed in 50 / DPHC. The expression level at 14 days of induction in 50 / DPHC was similar with that of vehicle control (Fig. 9C). In the case of Dcn mRNA expression, there was no difference between caffeine and DPHC on day 14 of adipogenic induction.

Lum mRNA was expressed in fully differentiated adipocyte which was induced from sADSCs (Fig. 10A). Caffeine suppressed the expression of Lum mRNA during induction and fully differentiated adipocyte (Fig. 10B). 0.4 / , $2 /$, and 10 / DPHC suppressed the expression of Lum mRNA expression during induction and fully differentiated adipocytes. In the case of 50 / DPHC, its expression was significantly suppressed during induction but was significantly increased in fully differentiated adipocytes (Fig. 10C). The expression levels of Lum mRNA were significantly higher in 50 / DPHC than in caffeine groups (Fig. 10).
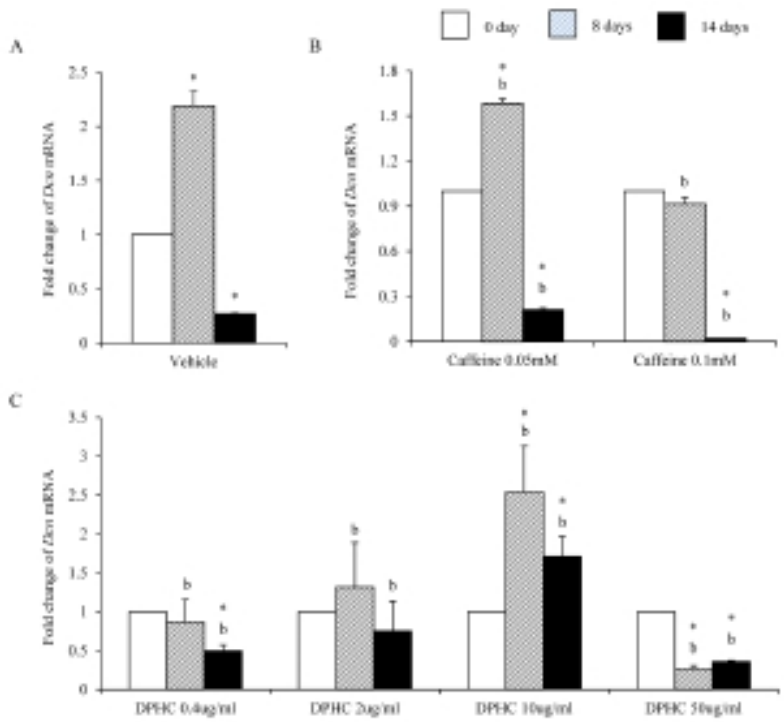

Fig. 9. Effects of caffeine and diphlorethohydroxycarmalol (DPHC) of decorin gene (Dcn). Mouse subcutaneous adipose derived stem cells (msADSCs) were incubated until 90-95\% confluency and then induced to adipocyte. Total RNAs were isolated at 0 day, 8 day, and 14 day of induction. To evaluate the change of the expression levels of $D c n$ mRNA, real-time PCR method was employed. *: $P<0.05$ control vs. caffeine or DPHC.
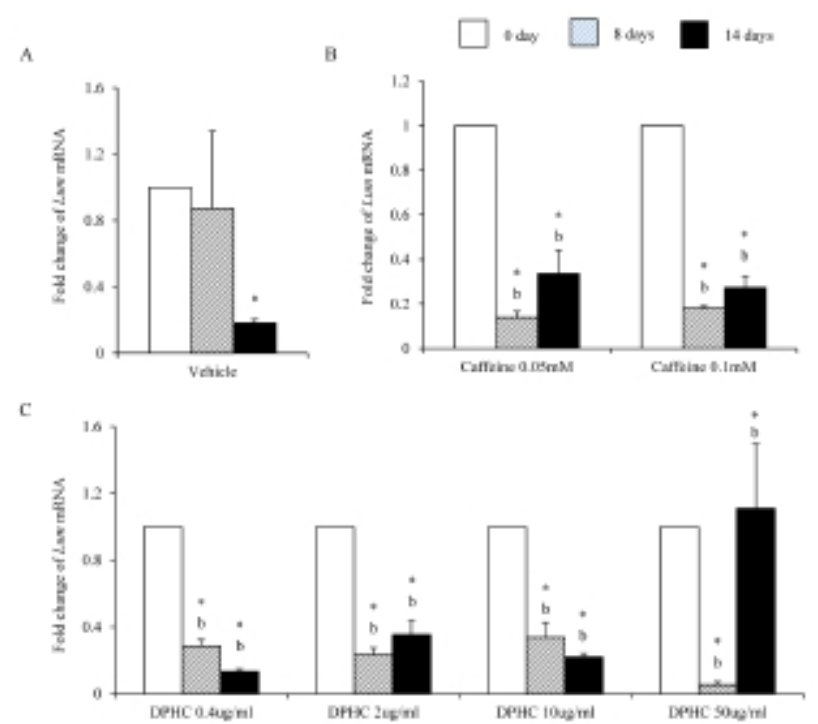

Fig. 10. Effects of caffeine and diphlorethohydroxycarmalol (DPHC) of lumican gene (Lum). Mouse subcutaneous adipose derived stem cells (msADSCs) were incubated until 90 $-95 \%$ confluency and then induced to adipocyte. Total RNAs were isolated at 0 day, 8 day, and 14 day of induction. To evaluate the change of the expression levels of Lum mRNA, real-time PCR method was employed. *: $P<0.05$ control vs. caffeine or DPHC. 


\section{DISCUSSION}

Growth and increase the number of adipocyte is one of the preferred origin of cellulite with gender specific dimorphic skin architecture, altered connective tissue septae (architecture), and microvascular alterations (de la Casa Almeida et al., 2012; Pavicic et al., 2006). Specific combinations of expressed ECM proteins are present in each adipose tissue and the expressions of these genes are related with the stability of adipose tissues. It is suggested that disturbances of the expression of ECM genes of adipose tissues are cause of disformation of skin such as cellulite. ECM has many key roles during tissue genesis and the changing of ECM construction means the change of cell shape, structure, or function in their sites of tissue. The change of female hypodermal connective tissue as being arranged radially or perpendicular to the skin surface is observed in cellulite area of pregnant women (Pierard \& Nizet, 2000; Smalls et al., 2006). The charicteristics of these histological changes are forming 'standing fat-cell chambers' in the surface layer of subcutaneous separated by connective tissue septa (de la Casa Almeida et al., 2012; Nurnberger. 1981). Although there are many suggested etiology for cellulite, the change of extracellular fibrila during this histological changes is suggested as a key factor. The importance of ECM of adipocyte focused because it is important in adipose tissue genesis and pathophysiology of it. Therefore, to understand the disformation of integument, it is important to understand how the ECM of adipose tissue is modified during adipogenesis or pathological adipose tissues. As first step to understanding the change of ECM construction, we analyzed the expression of extracellular fibrils during adipogenesis of ADS cells.

To treat the cellulite, various plants such as Coffea arabica, Camellia sinensis, Fucus vesciculosus, Ruscus aculeatus, Ginkgo biloba, Cynara scolymus, Centella asiatica, ivy, horse chestnut, sweet clover, red grapes, and Rubiaceas have been used to get specific active ingredients. The physiological effects of the ingredients of these plants are including antiinflammation, antiedema, stimulating the burning of fat, antioxident, increasing vascular permeability, improving microcirculation, and reducing lipogenesis (Bertin et al., 2001; Hexsel et al., 2005; Lesser et al., 1999; Rossi \& Vergnanini, 2000). Some of them help to restore the normal structure of the subcutaneous tissue and acting as anti-free radials (Hexcel et al., 2005; Seiler et al., 1994).

Collagens 1 through VI localize in a normal wild type fat pad. In mature adipocytes, collagens I, IV, and VI are abundantly expressed (Nakajima et al., 1998), while collagens II and III has considerably lower expression levels in adipocytes (Khan et al., 2009). In the adipose tissue of ob/ob and the $\mathrm{db} / \mathrm{db}$ mice, the expressions of ECM genes such as collagen VI are highly elevated (Khan et al., 2009). Preadipocytes synthesize ECM componets in the subcutaneous adipose tissue of obese human after treatment of proinflammatory mediators (Henegar et al., 2008). In this study, we found that the expressions of Col1a1, Col3a1, and Col6a1 were decreased during adipogenic induction and after differentiation of sADSC. On day 14 of induction the adipocyte (fully differentiated adipocyte) expressed significantly low level mRNA of Col1a1 and Col6a1. The reasons of the difference of that of Nakajima et al. (1998) may be the difference the stage of induction. On the other hand, the previous report, type 1 collagen is a basic substance of scaffold for tissue engineering with ASCs (Lu et al., 2007) can be supported by these results. In the case of collagen VI, it is known a hallmark of dysregulated adipose tissue in human and mouse. Col6a3 expression is significantly increased in both the abdominal and gluteal subcutaneous adipose (Khan et al., 2009). As showed in the Results, the expression levels of col6a1 mRNA was significantly decreased during adipogenesis. Interestingly caffeine also suppressed the expressions of Col1a1, Col3a1, and Col6a1. In addition, DPHC inhibited the expression of these collagens during induction of adipogenesis and fully differentiated adipocytes. The expression levels of Col6a1 mRNA were lower than that of caffeine in all concentration groups. Such results mean that DPHC may have more effects 
than caffeine on regulation of ECM during adipogenesis and on suppression the abnormality of ECM structure.

It is suggested that collagen type IV is synthesized during mesenchymal stem cell-adipocyte differentiation. And remodeling become necessary when intracellular accumulation of fat necessitates a dynamically supporting and instructive, partly denatured adipogenic pericellular type IV collagen scaffold (Silat et al., 2012). As expected our data support such a change of collagen type IV during adipogenesis. The expressions of Col2a1 and Col4a1 were increased during adipogenesis of scADSC. In the case of caffeine, it suppressed the expression of these collagens in $0.1 \mathrm{mM}$ concentration but it increased significantly the expression in $0.05 \mathrm{mM}$ concentration in fully differentiated adipocytes. In the case of DPHC, the expression of Col2a1 mRNA was significantly decreased by adipogenesis in all groups except 50 / group. Caffeine and DPHC stimulated the expression of Col4a1 mRNA in the specific concentration. It is suspected that DPHC is can be used in suppress the adipogenesis in a high concentration. Based on them it is suggested that collagen type II may be increas the expression levels during adipogenesis likes collagen type IV. It also suggested that DPHC has positive effects in normal adipose tissue generation.

Elastin allows resume the shape after stretching or contracting. The expression of Eln mRNA was significantly increased during adipogenesis but did not in fully differentiated adipocytes. Caffeine had not much effect on the expression of Eln during adipogenesis, but DPHC stimulated the expression of Eln in all concentration groups. Mfap5 protein is a component of the elastin-associated microfibrils. The change in Mfap5 mRNA expression during weight reduction correlated positively with the change of body fat mass (Vaittinen et al., 2011). In the case of msADSC, the expression levels of Mfap5 mRNA were decreased by adipogenesis induction. In the groups of caffeine and DPHC, the expression levels of Mfap5 mRNA were significantly lower than that of the vehicle control. However, it is unlike that the decrease of mRNA expression levels of Mfap5 mRNA is not depending on caffeine or DPHC.
Exceptionally high concentration of DPHC (50 / ) suppressed the expression of it in both adipogenic cells and fully differentiation adipocyte. Such results indicate that DPHC can be applied in keeping the stability of the ECM of adipose tissue.

Dermal glycosaminoglycans increased in cellulite area and serve greater water binding sites (Curri, 1993). The expression levels of biglycan correlates with inflammation of subcutaneous adipose tissue (Adapala et al., 2012). Biglycan is a leucin-rich repeat proteoglycan, consisted of protein core contaning leucin-rich repeat regions and two glycosaminoglycan (GAG) chains consisting of either chondroitin sulfate or dermatan sulfate. It found in the ECM of adipose tissue. Decorin is a leucin-rich proteoglycan (average $90-140 \mathrm{kD}$ ), consisted of a protein core containing leucine repeats with a glycosaminoglycan chain consisting of either chondroitin sulfate or dermatan sulfate. Decorin is closely related in structure to biglycan protein. They are a component of connective tissue, bind to type 1 collagen fibrils and play a role in matrix assembly. Lumican also is a leucine-rich proteoglycan. Lumian may regulate collagen fibril organization and circumferential growth, corneal transparency, and epithelial cell migration and tissue repair. As previously known, during adipogenesis of msADSC, the expression of Bgn mRNA, Dcn mRNA and Lum mRNA were decreased. The expression of lumican increases significantly in the adipose tissue of gestational diabetic mellitus (Oliva et al., 2013). In the case of decorin and biglycan, co-treatment of them with the proteoglycan fibronectin improves proliferation of preadipocytes (Ward \& Ajuwon, 2011). It is also known that biglycan activated inflammatory pathway in adipose tissue (Adapala et al., 2012). Interestingly caffeine and 50 / DPHC suppressed the expressions of Bgn mRNA and Don mRNA. The expression of Lum mRNA was suppressed by caffeine and DPHC $(\sim 10, /)$ but not in 50 / DPHC. It means that DPHC may involve in the stability of adipogenesis in SADSC. Put together our results, it is suggested that DPHC can be applied to suppress the inflammation in malignant adipose tissue 
and regulate the ECM during adipogenesis.

From this study, it is revealed that the cells which is under the differentiation to adipocyte in vitro, express ECM fibrils such as collagens, elastin, and glycoproteins in an induction time dependent manners. So far the main reason for using the plants extracts such as caffeine is to reduce fat deposit, but precisely they are it is depends on a number of factors. In addition to the previously suggested roles of DPHC derived from the extracts of $I$. okamurae such as antioxidant, suppressor of hyperglycemia (inhibitor of $\alpha$-glucosidase and $\alpha$-amylase), and antiinflamation, based on this study, we reveal firstly that the effects of DPHC on the expression of collagens, elastin, and glycoproteins during adipogenesis of sADSCs. Those results suggest followings: 1) DPHC may have antiadipogenic effect and has more effects than caffeine on regulation of ECM during adipogenesis. 2) DPHC has positive effects on normal adipose tissue generation and work as suppressor the abnormality of ECM structure. Such results indicate that DPHC can be applied in keeping the stability of the ECM of adipogenic tissues. In addition, it is expected that DPHC may help to restore the normal structure of the subcutaneous adipose tissue.

\section{ACK NOWLEDGEMENTS}

We thanks to the supporte of KICOX to (2012).

\section{REFERENCES}

Adapala VJ, Ward M, Ajuwon KM (2012) Adipose tissue biglycan as a potential anti-inflammatory target of sodium salicylate in mice fed a high fat diet. J Inflamm 9:15.

Agache P, Ovide MT, Laurent R (1976) Mechanical factors in striae distensae. In: Moretti GR, Rebora A (ed), Striae Distensae, Symposium Publication Division Brocades, Milan, Italy, pp 87-96.

Babelova A, Moreth K, Tsalastra-Greul W, Zeng-Brouwers J, Eickelberg O, Young MF, Bruckner P, Pfeilschifter
J, Schaefer RM, Gröne HJ, Schaefer L (2009) Biglycan, a danger signal that activates the NLRP3 inflammasome via toll-like and P2X receptors. J Biol Chem 284: 24035-24048.

Bertin C, Zunino H, Pittet JC, Beau P, Pineau P, Massonneau M, Robert C, Hopkins J (2001) A double-blind evaluation of the activity of an anti-cellulite product containing retinol, caffeine, and ruscogenine by a combination of several non-invasive methods. J Cosmet Sci 52:199210.

Bray GA (1989) Obesity: basic considerations and clinical approaches. Dis Mon 35:451-528.

Cao R, Brakenhielm E, Wahlestedt C, Thyberg J, Cao Y (2001) Leptin induces vascular permeability and synergistically stimulates angiogenesis with FGF-2 and VEGF. Proc Natl Acad Sci USA 98:6390-6395.

Chavey C, Mari B, Monthouel MN, Bonnafous S, Anglard P, Van Obberghen E, Tartare-Deckert S (2003) Matrix metalloproteinases are differentially expressed in adipose tissue during obesity and modulate adipocyte differentiation. J Biol Chem 278:11888-11896.

Chiellini C, Costa M, Novelli SE, Amri EZ, Benzi L, Bertacca A, Chhen P, Del Prato S, Friedman JM, Maffei M (2003) Identification of cathepsin $K$ as a novel marker of adiposity in white adipose tissue. J Cell Physiol 195:309-321.

Chun TH, Hotary KB, Sabeh F, Saltiel AR, Allen ED, Weiss SJ (2006) A pericellular collagenase directs the 3-dimensional development of white adipose tissue. Cell 125:577-591.

Cinti S (2001) The adipose organ: morphological perspectives of adipose tissues. Proc Nutr Soc 60:319-328.

Curri SB (1983) Aspetti biochimici. In: Ribuffo A, Bartoletti CA (ed), La Cellulite, Salus, Rome, pp 29-36.

Curri SB (1993) Cellulite and fatty tissue microcirculation. Cosmet Toilet 108:51-58.

de la Casa Almeida M, Suarez Serrano C, Rebollo Roldán J, Jiménez Rejano JJ (2012) Cellulite's aetiology: a review. J Eur Acad Dermatol Venereol doi: 10.1111/ j.1468-3083.2012.04622.x [epub ahead of print]. 
Gesta S, Bluher M, Yamamoto Y, Norris AW, Berndt J, Kralisch S, Boucher J, Lewis C, Kahn CR (2006) Evidence for a role of developmental genes in the origin of obesity and body fat distribution. Proc Natl Acad Sci USA 103:6676-6681.

Hausman GJ, Richardson RL (2004) Adipose tissue angiogenesis. J Anim Sci 82:925-934.

Henegar C, Tordjman J, Achard V, Lacasa D, Cremer I, Guerre-Millo M, Poitou C, Basdevant A, Stich V, Viguerie N, Langin D, Bedossa P, Zucker JD, Clement K (2008) Adipose tissue transcriptomic signature highlights the pathological relevance of extracellular matrix in human obesity. Genome Biol 21:9. R14.

Heo SJ, Hwang JY, Choi JI, Han JS, Kim HJ, Jeon YJ (2009) Diphlorethohydroxycarmalol isolated from Ishige okamurae, a brown algae, a potent $\alpha$-glucosidase and $\alpha$-amylase inhibitor, alleviates postprandial hyperglycemia in diabetic mice. Eur J Pharmacol 615:252-256.

Hexsel D, Orlandi C, Zechmeister do Prado D (2005) Botanical extracts used in the treatment of cellulite. Dermatol Surg 31:866-872.

Kang GJ, Han SC, Koh YS, Kang HK, Jeon YJ, Yoo ES (2012) Diphlorethohydroxycarmalol, isolated from Ishige okamurae, increases prostaglandin E2 throgh the expression of cyclooxygenase-1 and -2 in HaCaT human keratinocytes. Biomol Ther 20:520-525.

Khan T, Muise ES, Iyengar P, Wang ZV, Chandalia M, Abate N, Zhang BB, Bonaldo P, Chua S, Scherer PE (2009) Metabolic dysregulation and adipose tissue fibrosis: Role of collagen VI. Mol Cell Biol 29:15751591.

Khan MH, Victor F, Rao B, Sadick NS (2010) Treatment of cellulite: Part II. Advances and controversies. J Am Acad Dermatol 62:373-384.

Lesser T, Ritvo E, Moy LS (1999) Modification of subcutaneous adipose tissue by a methylxanthine formulation: a double-blind controlled study. Dermatol Surg 25:445462.

Lu F, Gao JH, Mizuro H, Ogawa R, Hyakusoku H (2007) Experimental study of adipose tissue differentiation using adipose-derived stem cells harvested from GFP transgenic mice. Zhoghua Zheng Xing Wai ke Za Zhi 23:412-416.

Milani GB, Natal Filho A, Amado João SM (2008) Correlation between lumbar lordosis angle and degree of gynoid lipodystrophy (cellulite) in asymptomatic women. Clinics 63:503-508.

Mirrashed F, Sharp JC, Krause V, Morgan J, Tomanek B (2004) Pilot study of dermal and subcutaneous fat structures by MRI in individuals who differ in gender, BMI, and cellulite grading. Skin Res Technol 10:161-168.

Nakajima I, Yamaguchi T, Ozutsumi K, Aso H (1998) Adipose tissue extracellular matrix: newly organized by adipocytes during differentiation. Differentiation 63:193-200.

Nurnberger F (1981) Practically important diseases of the subcutaneous fatty tissue (including so-called cellulite). Med Welt 32:682-688.

Oliva K, Barker G, Rice GE, Bailey MJ, Lappas M 2013. 2D-DIGE to identify proteins associated with gestational dibetes in omental adipose tissue. J Endocrinol 218:165-176.

Ortonne JP, Zartarian M, Verschoore M, Queille-Roussel C, Duteil L (2008) Cellulite and skin ageing: is there any interaction? J Eur Acad Dermatol Venereol 22: 827-834.

Park MH, Jeon YJ, Kim HJ, Han JS (2013) Effect of diphlorethohydroxycarmalol isolated from Ishige okamurae on apoptosis in 3T3-L1 preadipocytes. Phytother Res 27:931-936.

Pavicic T, Borelli C, Korting HC (2006) Cellulite-the greatest skin problem in healthy people? An approach. J Dtsch Dermatol Ges 4:861-870.

Perrini S, Laviola L, Cignarelli A, Melchiorre M, De Stefano F, Caccioppoli C, Natalicchio A, Orlando MR, Garruti G, De Fazio M, Catalano G, Memeo V, Giorgino R, Giorgino F (2008) Fat depot-related differenes in gene expression, adiponectin secretion, and insulin action and signalling in human adipocytes differentiated in vitro from precursor stromal cells. Diabetologia 51:155- 
164.

Pierard GE, Nizet JL, Pierard-Franchimont C (2000) Cellulite: from standing fat herniation to hypodermal stretch marks. Am J Dermatopathol 22:34-37.

Rossi AB, Vergnanini AL (2000) Cellulite: a review. J Eur Acad Dermatol Venereol 14:251-262.

Seiler M, Orecchioni AM, Vaution C (1994) Vesicular systems and multiple emulsions in cosmetology. In: Baran R, Maibach HI (ed), Cosmetic Dermatology, Williams \& Wilkins, Baltimore, pp 27-35.

Smalls LK, Hicks M, Passeretti D, Gersin K, Kitzmiller WJ, Bakhsh A, Wickett RR, Whitestone J, Visscher
MO (2006) Effect of weight loss on cellulite: gynoid lypodystrophy. Plast Reconstr Surg 118:510-516.

Vaittinen M, Kolehmainen M, Schwab U, Uusitupa M, Pulkkinen L (2011) Microfibrillar-associated protein 5 is linked with markers of obesity-relate extracellular matrix remodeling and inflammation. Nutr Diabetes 1:e15.

Ward M, Ajuwon KM (2011) Regulation of pre-adipocyte proliferation and apoptosis by the smal leucine-rich proteoglycans, biglycan and dicorin. Cell Prolif 44:343351. 